\title{
Trends in the Diagnosis and Treatment of Patients with Medullary Thyroid Carcinoma in Korea
}

\author{
Hwa Young Ahn ${ }^{1}$, Jae Eun Chae ${ }^{2}$, Hyemi Moon², Junghyun Noh³, Young Joo Park ${ }^{4}$, Sin Gon Kim ${ }^{5}$ \\ ${ }^{1}$ Department of Internal Medicine, Chung-Ang University College of Medicine; ${ }^{2}$ Department of Biostatistics, Korea University \\ College of Medicine, Seoul; ${ }^{3}$ Division of Endocrinology and Metabolism, Department of Internal Medicine, Inje University \\ Ilsan Paik Hospital, Inje University College of Medicine, Goyang; ${ }^{4}$ Department of Internal Medicine, Seoul National University \\ College of Medicine; ${ }^{5}$ Department of Internal Medicine, Korea University College of Medicine, Seoul, Korea
}

Background: Thyroid cancer is becoming increasingly common worldwide, but little is known about the epidemiology of medullary thyroid carcinoma (MTC). This study investigated the current status of the incidence and treatment of MTC using Korean National Health Insurance Service (NHIS) data for the entire Korean population from 2004 to 2016.

Methods: This study included 1,790 MTC patients identified from the NHIS database.

Results: The age-standardized incidence rate showed a slightly decreasing or stationary trend during the period, from 0.25 per 100,000 persons in 2004 to 0.19 in 2016. The average proportion of MTC among all thyroid cancers was $0.5 \%$. For initial surgical treatment, $65.4 \%$ of patients underwent total thyroidectomy. After surgery, external-beam radiation therapy (EBRT) was performed in $10 \%$ of patients, a proportion that increased from $6.7 \%$ in 2004 to $11.0 \%$ in 2016 . Reoperations were performed in $2.7 \%$ of patients $(n=49)$ at a median of 1.9 years of follow-up (interquartile range, 1.2 to 3.4). Since November 2015, $25(1.4 \%)$ patients with MTC were prescribed vandetanib by December 2016.

Conclusion: The incidence of MTC decreased slightly with time, and the proportion of patients who underwent total thyroidectomy was about $65 \%$. EBRT, reoperation, and tyrosine kinase inhibitor therapy are additional treatments after initial surgery for advanced MTC in Korea.

Keywords: Epidemiology; Incidence; Thyroid cancer, medullary; Therapeutics

\section{INTRODUCTION}

Medullary thyroid carcinoma (MTC) originates from parafollicular C-cells; therefore, its treatment and prognosis are different

Received: 13 May 2020, Revised: 26 August 2020,

Accepted: 22 September 2020

Corresponding authors: Young Joo Park

Department of Internal Medicine, Seoul National University College of

Medicine, 101 Daehak-ro, Jongno-gu, Seoul 03080, Korea

Tel: +82-2-2072-4183, Fax: +82-2-762-2199, E-mail: yjparkmd@snu.ac.kr

Sin Gon Kim

Division of Endocrinology and Metabolism, Department of Internal Medicine, Korea University College of Medicine, 73 Goryeodae-ro, Seongbuk-gu, Seoul 02841, Korea

Tel: +82-2-920-5890, Fax: +82-2-922-5974, E-mail: k50367@korea.ac.kr from those of other thyroid cancers, which are driven by follicular cells [1-4].

According to the Surveillance, Epidemiology, and End Results Program (SEER) database (1975 to 2016), MTC accounted for

\section{Copyright $\odot 2020$ Korean Endocrine Society}

This is an Open Access article distributed under the terms of the Creative Commons Attribution Non-Commercial License (https://creativecommons.org/ licenses/by-nc/4.0/) which permits unrestricted non-commercial use, distribution, and reproduction in any medium, provided the original work is properly cited. 
$1.6 \%$ of all thyroid cancer in all races, $1.6 \%$ in Whites, $1.8 \%$ in Blacks, and $0.9 \%$ in Asian/Pacific Islanders [5]. However, limited data are available regarding the nationwide proportion of MTC in Asian countries including Korea; the only relevant study, which analyzed data from the Korea National Cancer Incidence Database during 1997 to 2011, reported that MTC accounted for $0.6 \%$ of cases of thyroid cancer in Korea [6]. In addition, because of the rapid worldwide increment in the incidence of papillary thyroid cancer [7,8], which has been particularly noticeable in Korea due to the increasing rate of papillary thyroid microcarcinoma [9], the incidence of MTC might have changed. In addition, a recent paper analyzing the SEER database from 1983 to 2012 showed that the incidence of medullary thyroid microcarcinoma (micro-MTC), which is less than $1 \mathrm{~cm}$, increased with time [10]. These results suggest that MTC could be found at an early stage due to the increasing frequency of screening tests using thyroid ultrasonography, and the incidence of MTC has correspondingly increased in national data from the United States [10], Canada [11], and Norway [12]. In contrast, the incidence trends of MTC were stable in China [13] and England [14].

Regarding the prognosis of MTC, the mortality rate has been reported to range from $13.5 \%$ to $15.6 \%[1,15]$. The likelihood of survival depends on the patient's disease status and whether any persistent or recurrent tumor exists; furthermore, different survival rates have been reported in patients according to whether they had locoregional or distant metastasis [10]. Patients with tumors confined to the thyroid gland had an excellent 10 -year survival rate of $95.6 \%$, whereas those with regional-stage disease had a rate of $75.5 \%$. Moreover, only $40 \%$ of patients with distant metastases at diagnosis survived for 10 years [16]. However, the overall thyroid cancer mortality rate in Korea has decreased from 1985 to 2010 [17] and the reduction in mortality from thyroid cancer has also been confirmed in global data [18]. Therefore, the prognosis of MTC may have changed in recent decades according to the changes in its incidence or likelihood of being detected at an early stage. A recent study of Korean MTC patients showed that the recurrence rate had decreased over time, concomitantly with reductions in tumor size and the extent of extrathyroidal invasion [19]. However, according to the results of SEER data analysis, despite an increase in the diagnosis of micro-MTC [20], the rate of total thyroidectomy and lymph node dissection has increased in recent years [10]. However, when the disease-specific survival rates at 5 and 10 years were compared by dividing the 30-year intervals into 1983-1992, 1993-2002, and 2003-2012, there was no difference in the survival rates in the SEER database [10].
In order to provide proper, up-to-date guidelines about the treatment and follow-up of MTC patients, it is important to have exact information on the current status of MTC diagnosis, treatment, and prognosis. For this purpose, this study investigated the current status of incidence and treatment of MTC using Korean National Health Insurance Service (NHIS) data for the whole Korean population from 2004 to 2016.

\section{METHODS}

\section{Data source and study population}

This study is based on the Korean NHIS database [21], which covers information about the medical use of $97 \%$ of the Korean population. All outpatient and inpatient data related to diagnosis and treatment costs are submitted to the NHIS by hospitals in Korea in order to claim payments for patient care.

Because NHIS data since 2002 can be extracted, the present study analyzed data from 2004 to 2016, with a 2-year wash-out period. We used International Classification of Disease, 10th edition (ICD-10) disease codes [22]. Specifically, the disease codes used in this study were those for thyroid cancer (C73), hyperparathyroidism or parathyroid tumors (E21, D351, C75) and adrenal tumors (D350, D441, C74).

This study was approved by the Institutional Review Board of Chung-Ang University Hospital (IRB 1711-005-16115). The requirement for informed consent was waived.

\section{Definition of medullary thyroid cancer}

To identify MTC patients, we first selected all thyroid cancer patients using the ICD-10 code of C73 in the NHIS data, because the NHIS database contains no histologic information. Patients with thyroid cancer were defined as those who had two or more C73 codes during the study period (from 2004 to 2016) and who underwent thyroid surgery within 2 years of receiving the first C73 code. We also adapted a special exempted calculation code for the Korean health insurance system, which was developed in 2006 and has since been applied to all cancer patients at the time of initial diagnosis to provide additional support for medical costs. The number of patients extracted using the operational definition of newly-diagnosed thyroid cancer $(n=397,536)$ was compared with the number of thyroid cancer patients $(n=368,250)$ from the reports of the Korean National Cancer Center (https://ncc.re.kr/cancerStatsList.ncc?searchKey $=$ total $\&$ search Value $=\&$ pageNum $=1$ ).

Next, we excluded those who had two or more serum thyroglobulin measurements after surgery $(n=395,626)$, in order to 
exclude pure or co-existing follicular cell-driven thyroid cancers. Then, we defined MTC patients $(n=1,790)$ as those who had two or more serum calcitonin measurements after initial surgery (Supplemental Fig. S1). The proportion of MTC extracted by this operational definition was $0.5 \%$ of all cases of thyroid cancer, which is similar to the proportion of $0.6 \%$ reported in a survey on the status of thyroid cancer in Korea from 1997 to 2011 using the Korean National Cancer Center registry database [6]. In addition, the number of MTC patients was comparable with those collected from 2004 to 2012 at 12 hospitals in Korea [23,24].

\section{Definition of other components of multiple endocrine neoplasia present clinically}

MTC associated with multiple endocrine neoplasia (MEN) syndrome is classified as MEN type 2 (MEN2), which in turn is divided into two subtypes: MEN2A is accompanied by parathyroid adenoma ( $10 \%$ to $25 \%)$ or pheochromocytoma $(>50 \%)$ in addition to MTC, and MEN2B is accompanied by pheochromocytoma $(>50 \%)$ or other associated abnormalities such as mucosal neuroma, marfanoid habitus, or megacolon [25]. To examine whether MEN2 was present, we checked diagnoses of hyperparathyroidism or parathyroid tumor (E21, D351, C750) or the parathyroid surgery code (P4541-43), as well as codes for adrenal tumor diagnosis (D350, D441, C741, C749) or adrenal surgery (P4571, P4572).

\section{Definition of treatment}

Total thyroidectomy was defined as surgical codes P4552 and P4561, and lobectomy was confirmed by the presence of the codes P4551, P4553, and P4554. Reoperation due to recurrent or persistent tumors was defined as any thyroid surgery with or without lymph node dissection (P2112-4, P2116, P2118, P2119) for 6 months or more, or lymph node dissection alone performed at any time after the first thyroidectomy. Additional thyroid surgery with or without lymph node dissection within 6 months after the first operation was not considered as reoperation related to recurrence or persistence.

After the surgical treatment of MTC, additional treatment with external-beam radiation therapy (EBRT), radioactive iodine (RAI) therapy, or chemotherapy with a tyrosine kinase inhibitor (TKI) can be considered. For adjuvant treatment, EBRT was confirmed by the treatment codes HD061 and HZ271 and RAI was confirmed by the drug code for sodium iodide (I-131). Prescriptions of vandetanib were also found using the corresponding drug code in the inquiry system of Health Insurance Review and Assessment Service.
Postoperative hypothyroidism was defined by the presence of at least two levothyroxine prescriptions after surgery. Calcium prescriptions were also investigated.

\section{Statistical analysis}

The ratio of MTC in each year was expressed as the proportion of MTC per 1,000 cases of thyroid cancer. The age-standardized incidence rate was defined as the weighted average crude agespecific rate, where the crude rates were calculated for different age groups and the weights were the proportion of persons in the corresponding age groups of a standard population (2000) [26]. The annual percent change (APC) can be expressed as $\left(\exp ^{(b)}-1\right) \times 100$, where $b$ is the estimated slope from a linear regression on the logarithmic scaled age-standardized rate and calendar year. Categorical variables are presented as numbers and percentages. For statistical analysis, SAS software version 9.3 (SAS Institute Inc, Cary, NC, USA) was used.

\section{RESULTS}

\section{Trends in the age and sex distribution and incidence of} medullary thyroid cancer during 2004 to 2016

Among the 1,790 patients, 467 (26\%) were male and 1,323 (74\%) were female, and their mean age at diagnosis of MTC was 55.4土 12.5 years old. The majority of them (73\%) were between 40 and 69 years of age. The sex and age distribution did not change throughout the period (Fig. 1A, B). The age-standardized incidence rate (Fig. 1C) showed a slightly decreasing or stationary trend during the period, from 0.25 per 100,000 persons in 2004 to 0.19 per 100,000 persons in 2016 . The APC was $-2.4 \%$.

The average proportion of MTC was $0.5 \%$ (1,790 of 397,536) among all thyroid cancers. However, a sudden decrement was observed in 2008, as the number of cases of MTC per 1,000 total thyroid cancer cases (Fig. 1D) was about 7 to 8 from 2004 to 2007 and decreased to 3 to 4 starting in 2008. The number increased again to 5 from 2015.

\section{Proportions of other phenotypes of multiple endocrine neoplasia}

Among the phenotypes of MEN, the presence of co-morbid parathyroid adenomas (or hyperparathyroidism) and/or adrenal masses was investigated. A total of $40(2.2 \%)$ patients were diagnosed with one or two of the above conditions concurrently with or after the diagnosis of MTC, of whom eight (20\%) had a parathyroid adenoma, 31 (77\%) had an adrenal mass, and one (3\%) had both. 

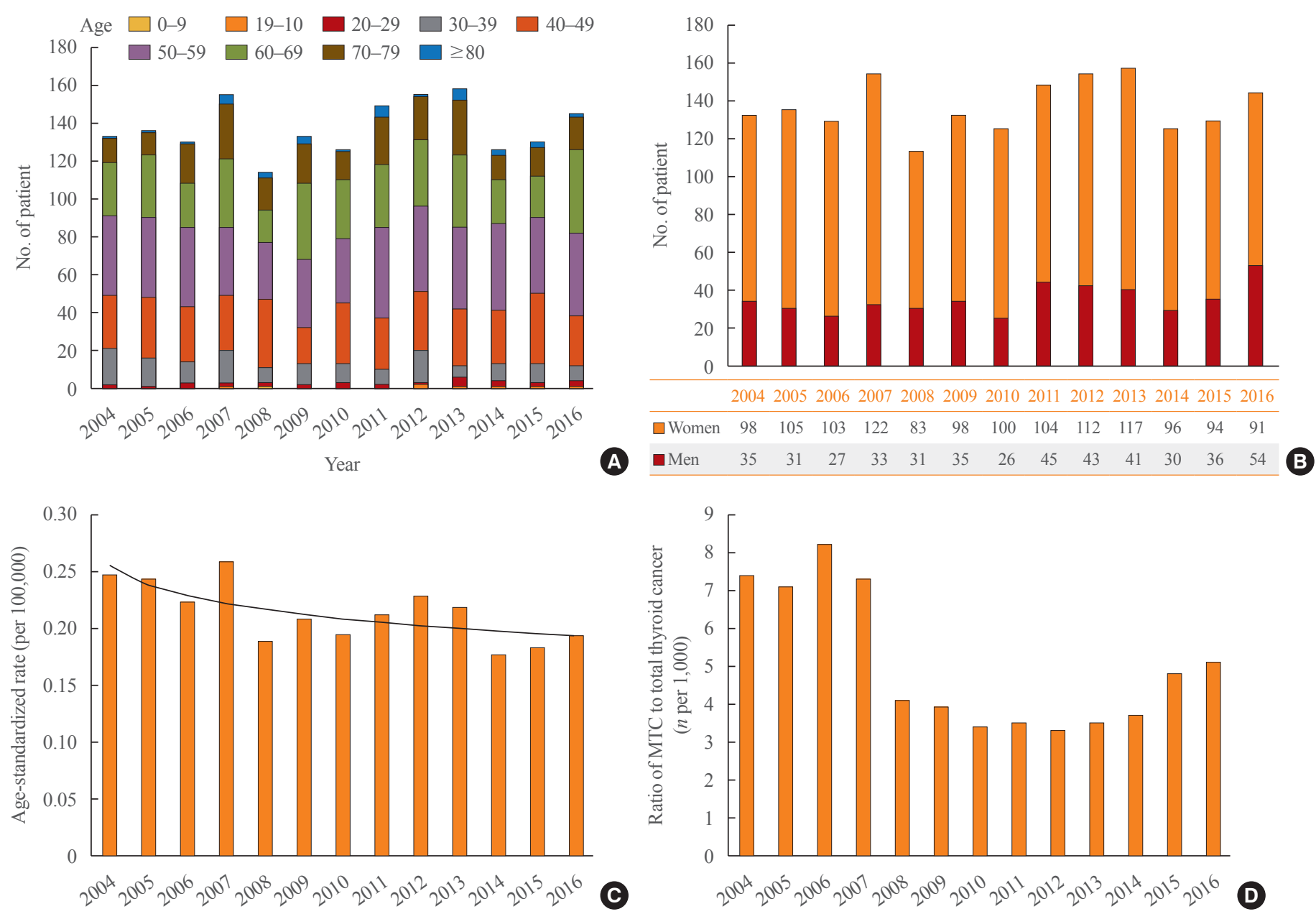

Fig. 1. (A) Number of patients with medullary thyroid cancer (MTC) according to age range by year. (B) Number of patients with MTC according to sex by year. (C) Age-standardized incidence rate of MTC by year. (D) Number of MTC patients per 1,000 total thyroid cancers by year.

Trends in the initial surgical extent and replacement of levothyroxine and calcium in 2004 to 2016

For initial surgical treatment, $65.4 \%$ of patients underwent total thyroidectomy and $34.6 \%$ of patients had lobectomy, and the proportion did not change during the period (Fig. 2A). The rate of completion thyroidectomy within 6 months from the first operation was 3.3\% among the patients who underwent lobectomy.

Thyroid hormone replacement was performed in $59.6 \%$ of the 598 patients who underwent lobectomy only. The percentage of patients who received calcium supplementation after thyroid surgery was $42.5 \%$.

\section{Radiation therapy after surgery}

After surgery, EBRT was performed in $10 \%$ of patients, and this proportion increased from $6.7 \%$ in 2004 to $11.0 \%$ in 2016 (Fig. 2B). Of these patients, $60 \%$ underwent EBRT within 1 year af- ter surgery, while the remaining $40 \%$ were treated with EBRT more than 1 year after surgery.

The overall proportion of patients who underwent RAI treatment was similar, at $7.5 \%$, but this proportion decreased dramatically over time, from $29.1 \%$ in 2004 to $0.8 \%$ in 2014 (Fig. 2C).

\section{Reoperation and prescription of tyrosine kinase inhibitors during follow-up}

Reoperation was performed in $2.7 \%(n=49)$ of patients at a median of 1.9 years of follow-up (interquartile range, 1.2 to 3.4). The average interval until reoperation was $2.7 \pm 2.3$ years from the first operation. Thirty-seven (75.5\%) reoperations were performed within the first 3 years, and 43 (87.8\%) occurred within 5 years (Fig. 3A). Of the patients who underwent lobectomy, five $(10.2 \%)$ were reoperated, and the remaining 44 patients $(89.8 \%)$ initially underwent total thyroidectomy. Of the 171 pa- 

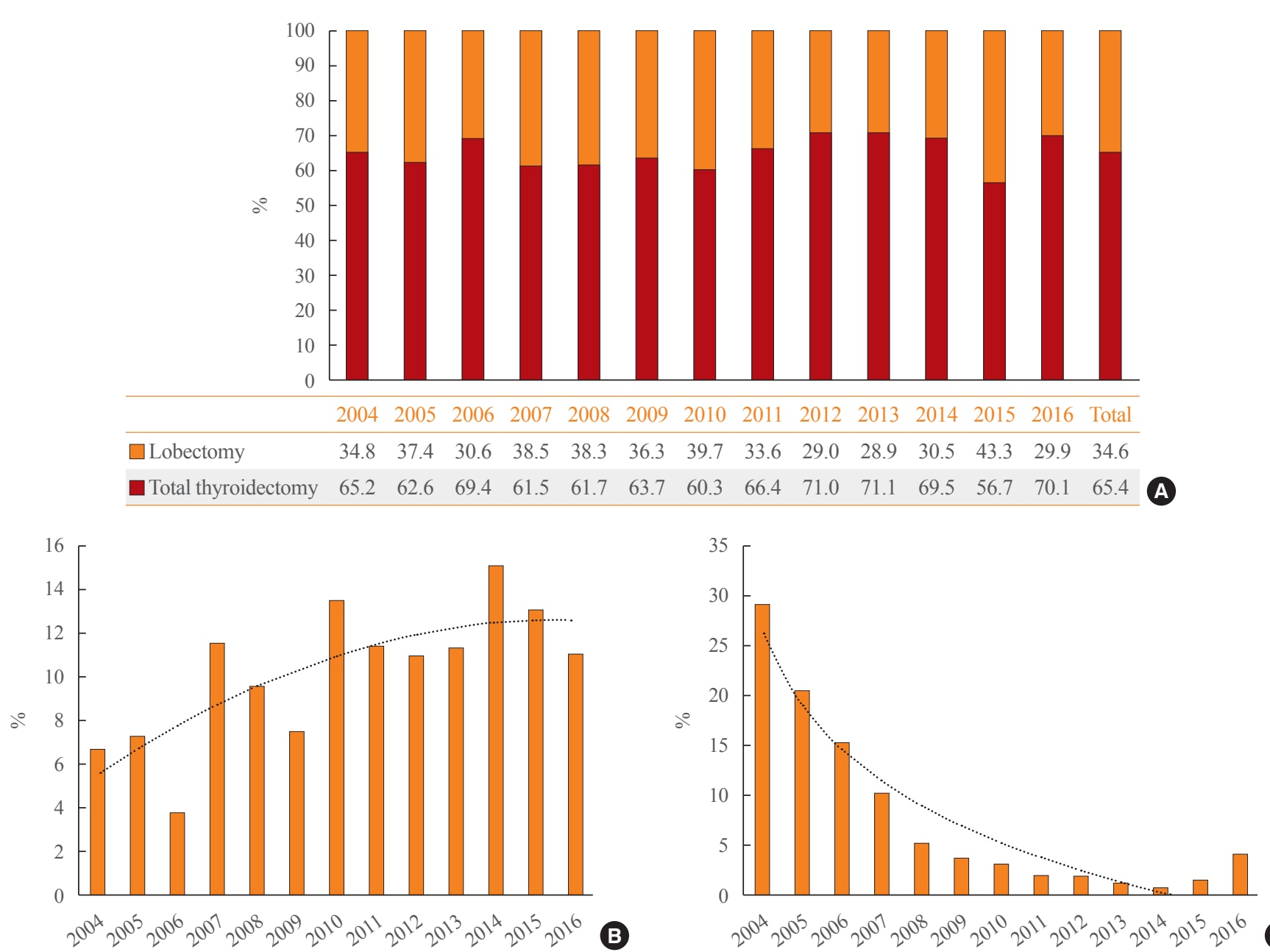

Fig. 2. (A) Percentage of patients who underwent thyroid surgery (total thyroidectomy and lobectomy) by year. (B) Percentage of patients who underwent external-beam radiation therapy by year. (C) Percentage of patients who underwent radioactive iodine treatment by year.

tients who underwent EBRT after surgery, seven (4.1\%) were reoperated (Fig. 3B).

In Korea, vandetanib has been prescribed since November 2015 for patients with advanced MTC. A total of 25 (1.4\%) patients with MTC were prescribed vandetanib by December 2016 . Six patients underwent reoperation, six patients received radiation therapy, and two patients underwent both reoperation and radiation therapy prior to the use of vandetanib (Fig. 3B). The average duration of vandetanib treatment was 199.7 days (range, 28 to 480). The mean age at the start of vandetanib was 50.4 years (range, 14 to 70). Six of the vandetanib-treated MTC patients died.

When we compared the rate of reoperation or vandetanib treatment according to radiation therapy, there was no statistically significant difference in the frequency of reoperation $(2.4 \%$ in the no-EBRT group vs. $3.8 \%$ in the EBRT group, $P=0.318$ ).
However, patients who received EBRT were significantly more likely to receive vandetanib treatment $(1.1 \%$ in the no-EBRT group vs. $4.3 \%$ in EBRT group, $P=0.002$ ) (Fig. 3C).

\section{DISCUSSION}

The age-standardized incidence ratio of MTC slightly decreased during 2004 to 2016 in Korea, with an APC of $-2.4 \%$. The proportion of MTC among total thyroid cancers showed a marked decrement of about $50 \%$ from 2008 to 2014 , but it slightly reincreased in 2015. Approximately two-thirds of patients underwent total thyroidectomy as an initial treatment, and this number did not meaningfully change during the time period. However, the proportion of patients who underwent ERBT gradually increased, while the proportion of patients who underwent RAI markedly decreased. 


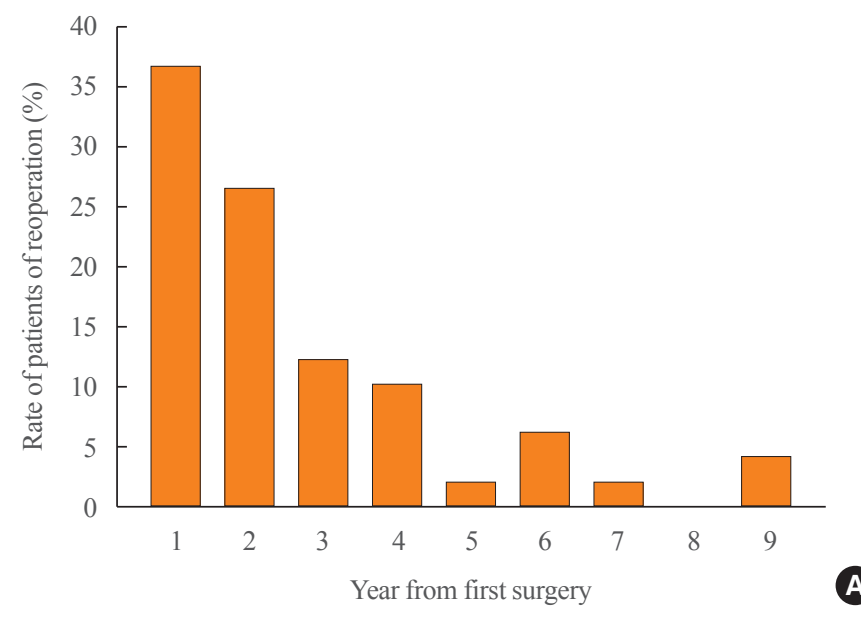

Initial surgery only $n=1,553$
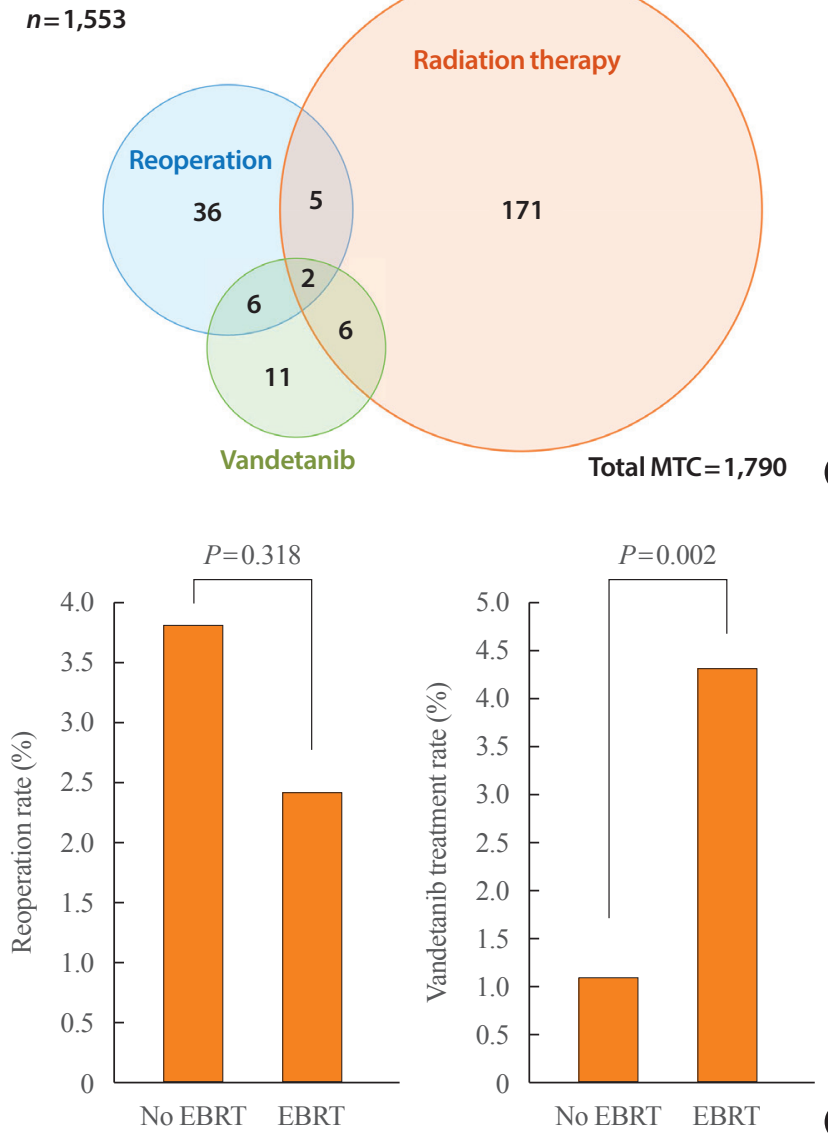

Fig. 3. (A) Distribution of the interval between the first surgery to the time of reoperation. (B) Number of medullary thyroid cancer (MTC) patients who underwent additional treatments after the first operation. (C) Difference in the rate of reoperation and vandetanib treatment depending on external-beam radiation therapy (EBRT).

The incidence of MTC showed different trends from the overall incidence of total thyroid cancer reported by the Korean Na- tional Cancer Center statistics [27], which primarily reflected trends in PTC. According to these statistics, the total incidence of thyroid cancer increased annually at a rate of $22.5 \%$ from 1999 to 2011, and then decreased by $-108 \%$ from 2011 to 2017. As shown in Fig. 1D, the ratio of MTC suddenly dropped to almost half compared to previous years. The reason for this is that the average diagnosis of differentiated thyroid cancer almost doubled since 2008 compared to 2004 to 2007 . However, the number of MTC diagnoses remained relatively constant; therefore, the ratio of MTC decreased starting in 2008. This change reflects the issue of overdiagnosis of thyroid cancer, which has been a major concern since 2014 in Korea. Regardless of the overall trends in thyroid cancer, however, the steadily decreasing APC of MTC in this study, although it was only $-2.4 \%$, suggests that the incidence of MTC is likely to have decreased or to have remained unchanged in Korea in the most recent decade. The average incidence of 0.19 to 0.25 per 100,000 population is similar to that reported in other countries: 0.18 per 100,000 population in the US SEER data (1983 to 2012) [7,10], 0.22 per 100,000 person-years in Norway (1994 to 2016) [12], and 0.19 per 100,000 person-years in Denmark (1997 to 2014) (Table 1) [28]. These suggest that the incidence of MTC is similar regardless of regions or ethnicity, unlike PTC (https://gco.iarc.fr/).

In terms of age at diagnosis of MTC, the patients in our study were found to be slightly older at the time of diagnosis, with an average age of 55 years, than those in previous studies, which reported an average age of 43 to 48 years $[29,30]$. However, in another study of Israeli patients, patients diagnosed from 2006 to 2016, with an average age of 54 years, were older than before 1995 (average of 38 years), showing similar results to our study [31] and national data from several other countries also reported that MTC was diagnosed at an average age of 52 to 55 years (Table 1).

Previous studies $[1,16,30]$ and other national epidemiological data (Table 1) have reported that the ratio of men and women among MTC patients is almost 1:1 to 1.5. However, a clearer female predominance of MTC was observed in our study, which found that three times more women than men developed MTC. This sex ratio is similar to that of PTC [7,32]. However, whether the risk of MTC is higher in Korean women than in those from other countries is still unclear, and future nationwide studies could clarify this result.

For the surgical treatment of MTC, the American Thyroid Association (ATA) guideline recommends total thyroidectomy and central neck dissection even if no lymph node metastasis is found [4]. In our results, however, only $65 \%$ of patients underwent to- 
Table 1. Comparison of Epidemiological Data on Medullary Thyroid Cancer in Several Nationwide Databases

\begin{tabular}{lcrcccccc}
\hline Study & Period & $\begin{array}{c}\text { No. of } \\
\text { patient }\end{array}$ & $\begin{array}{c}\text { Age standardized incidence } \\
\text { rate, } / 100,000 \text { person-year }\end{array}$ & $\begin{array}{c}\text { Annual percent } \\
\text { change }\end{array}$ & $\begin{array}{c}\text { Mean } \\
\text { age }\end{array}$ & $\begin{array}{c}\text { Sex ratio, } \\
\text { female:male }\end{array}$ & $\begin{array}{c}\text { Total thyroidectomy } \\
\text { rate, } \%\end{array}$ \\
\hline USA [7,10] & $1983-2012$ & 2,940 & 0.18 & 0.7 & 52.6 & $58.5: 41.5$ & 74.6 \\
Norway [12] & $1994-2016$ & 201 & 0.22 & 0.3 & 55.0 & $57: 43$ & 87.0 \\
Denmark [28] & $1997-2014$ & 224 & 0.19 & 1.0 & 52.4 & $58.9: 41.1$ & 91.0 \\
Present study (South Korea) & $2004-2016$ & 1,790 & 0.20 & -2.4 & 55.4 & $74: 26$ & 65.4 \\
\hline
\end{tabular}

tal thyroidectomy. In addition, the rate of completion was only $3.3 \%$. The reason why the rate of total thyroidectomy was lower than expected may have been that many nodules had an indeterminate result on fine-needle aspiration before surgery and a definitive diagnosis was made after lobectomy. The higher frequency of small MTC in Korea (31.1\% less than $1 \mathrm{~cm}, 35.4 \% 1$ to 2 $\mathrm{cm})$ [23], could be another possible explanation, because additional surgery is not needed if lymph node metastasis is not suspected in small MTC less than $1 \mathrm{~cm}$ in size [4]. In addition, the fact that about $81.1 \%$ to $85 \%$ of MTCs were sporadic in the most recent decade in Korea [23,33] could reduce the proportion of patients who underwent total thyroidectomy.

According to ATA guidelines for MTC, adjuvant EBRT should be considered in patients with a high risk of recurrence or with incompletely resected MTC [4]. However, RAI treatment is not recommended because it has been found to be ineffective in improving disease-specific survival in MTC patients [34]; instead, $\mathrm{RAI}$ is only recommended in MTC patients who also have PTC or FTC. The reason why RAI was performed in MTC patients is unclear, but it is possible that some patients with co-existing PTC or FTC could have been included, even though we tried to exclude them by using an operational definition based on serum thyroglobulin tests. Additionally, as the RAI treatment rate was mostly $20 \%$ to $30 \%$ in 2004 to 2005 , and decreased to less than $10 \%$ starting in 2007 , it is possible that many MTC patients received RAI treatment before 2006, because there were not yet well-established treatment guidelines for MTC treatment at that time. The trends of increasing EBRT and decreasing RAI over time also could be interpreted as reflecting a change in the treatment strategy for MTC according to recommendations in research and guidelines [4].

Six of the patients treated with vandetanib died; however, since data about relapse or persistent disease were not available, it was impossible to investigate the prognosis of MTC in this study. Reoperations were performed in $2.7 \%(n=49)$ of patients at a median of 1.9 years of follow-up (interquartile range, 1.2 to 3.4). Furthermore, $4.1 \%(n=74)$ of patients received EBRT more than
1 year after surgery and $1.4 \%(n=25)$ patients with MTC were prescribed vandetanib. Therefore, it can be estimated that 127 (7.1\%) patients received active treatment due to recurrent or persistent disease.

Interestingly, there was no statistically significant difference in the reoperation rate according to EBRT. However, patients who underwent EBRT were significantly more likely to receive vandetanib treatment (Fig. 3C). A possible reason for this difference is that it is more likely that surgery was not considered in these patients because of the technical difficulties and high complication rate of reoperation due to tissue damage at the site of EBRT. Further studies to clarify the long-term outcomes for each treatment after initial therapy are required.

This study has some limitation. First, because the ICD-10 codes do not include information about the histologic subtypes of thyroid cancer, an operational definition was used to select MTC patients. However, this method may be inaccurate compared to using diagnostic codes with histological subtypes. In addition, some non-expert physicians might not check serum thyroglobulin, or might check calcitonin instead of thyroglobulin, during follow-up in real-world practice; therefore, some patients with follicular cell-driven thyroid carcinoma might have been included in the group of MTC patients, or the number of MTC patients may have been underestimated. Secondly, since only data billed to the National Health Insurance were checked, no information was available on the results of blood tests, RET mutations, postoperative pathological results (stage, tumor size, lymph node metastasis, etc.), or changes in calcitonin levels. Third, for MEN diagnosis, the proportion of patients with a pheochromocytoma diagnosis may not have been accurate because biochemical or pathology results could not be confirmed. Therefore, the proportion of patients with MEN may have been overestimated due to the inclusion of non-functioning adrenal adenoma, or underestimated because there may have been some cases of pheochromocytoma that were not diagnosed due to the relatively short follow-up period.

However, an advantage of this study is that it included infor- 
mation on MTC diagnosis and treatment for almost the entire population of Korea.

In conclusion, the incidence of MTC decreased over time, and the rate of total thyroidectomy was about $65 \%$. External radiation therapy is on the rise, and a new treatment, TKI therapy (vandetanib), was found to be prescribed in patients with advanced MTC in Korea.

\section{CONFLICTS OF INTEREST}

No potential conflict of interest relevant to this article was reported.

\section{ACKNOWLEDGMENTS}

This research was supported by a grant from the Korea Health Technology R\&D Project through the Korea Health Industry Development Institute (KNIDI), funded by the Ministry of Health and Welfare, Republic of Korea (grant number: HI17C1926). We would like to thank the Committee of Clinical Practice Guidelines of the Korean Endocrine Society.

\section{AUTHOR CONTRIBUTIONS}

Conception or design: H.Y.A., J.N., Y.J.P. Acquisition, analysis, or interpretation of data: H.Y.A., J.E.C., H.M., S.G.K. Drafting the work or revising: H.Y.A., Y.J.P., S.G.K. Final approval of the manuscript: H.Y.A., Y.J.P.

\section{ORCID}

Hwa Young Ahn https://orcid.org/0000-0002-2737-6759

Young Joo Park https://orcid.org/0000-0002-3671-6364

Sin Gon Kim https://orcid.org/0000-0002-7430-3675

\section{REFERENCES}

1. Kebebew E, Ituarte PH, Siperstein AE, Duh QY, Clark OH. Medullary thyroid carcinoma: clinical characteristics, treatment, prognostic factors, and a comparison of staging systems. Cancer 2000;88:1139-48.

2. Faik Erdogan M, Gursoy A, Erdogan G, Kamel N. Radioactive iodine treatment in medullary thyroid carcinoma. Nucl Med Commun 2006;27:359-62.

3. Schwartz DL, Rana V, Shaw S, Yazbeck C, Ang KK, Morrison $\mathrm{WH}$, et al. Postoperative radiotherapy for advanced

818 www.e-enm.org medullary thyroid cancer: local disease control in the modern era. Head Neck 2008;30:883-8.

4. Wells SA Jr, Asa SL, Dralle H, Elisei R, Evans DB, Gagel RF, et al. Revised American Thyroid Association guidelines for the management of medullary thyroid carcinoma. Thyroid 2015;25:567-610.

5. Howlader N, Noone AM, Krapcho M, Miller D, Brest A, Yu M, et al. SEER Cancer Statistics Review, 1975-2016, National Cancer Institute [Internet]. Bethesda: SEER; 2019 [cited 2020 Oct 19]. Available from: https://seer.cancer.gov/ csr/1975_2016/.

6. Oh CM, Jung KW, Won YJ, Shin A, Kong HJ, Lee JS. Ageperiod-cohort analysis of thyroid cancer incidence in Korea. Cancer Res Treat 2015;47:362-9.

7. Lim H, Devesa SS, Sosa JA, Check D, Kitahara CM. Trends in thyroid cancer incidence and mortality in the United States, 1974-2013. JAMA 2017;317:1338-48.

8. Sanabria A, Kowalski LP, Shah JP, Nixon IJ, Angelos P, Williams MD, et al. Growing incidence of thyroid carcinoma in recent years: factors underlying overdiagnosis. Head Neck 2018;40:855-66.

9. Park S, Oh CM, Cho H, Lee JY, Jung KW, Jun JK, et al. Association between screening and the thyroid cancer "epidemic" in South Korea: evidence from a nationwide study. BMJ 2016;355:i5745.

10. Randle RW, Balentine CJ, Leverson GE, Havlena JA, Sippel RS, Schneider DF, et al. Trends in the presentation, treatment, and survival of patients with medullary thyroid cancer over the past 30 years. Surgery 2017;161:137-46.

11. Ellison LF, Bushnik T. Changing trends in thyroid cancer incidence in Canada: a histologic examination, 1992 to 2016. Health Rep 2020;31:15-25.

12. Opsahl EM, Akslen LA, Schlichting E, Aas T, Brauckhoff K, Hagen AI, et al. Trends in diagnostics, surgical treatment, and prognostic factors for outcomes in medullary thyroid carcinoma in Norway: a nationwide population-based study. Eur Thyroid J 2019;8:31-40.

13. Du L, Wang Y, Sun X, Li H, Geng X, Ge M, et al. Thyroid cancer: trends in incidence, mortality and clinical-pathological patterns in Zhejiang Province, Southeast China. BMC Cancer 2018;18:291.

14. National Cancer Registration and Analysis Service. Thyroid cancer: trends by sex, age and histological type [Internet]. London: National Cancer Intelligence Network; c2010 [cited 2020 Oct 19]. Available from: http://www.ncin.org.uk/ publications/data_briefings/thyroid_cancer_trends_by_sex_

Copyright (C) 2020 Korean Endocrine Society 
age_and_histological_type.

15. Rendl G, Manzl M, Hitzl W, Sungler P, Pirich C. Long-term prognosis of medullary thyroid carcinoma. Clin Endocrinol (Oxf) 2008;69:497-505.

16. Roman S, Lin R, Sosa JA. Prognosis of medullary thyroid carcinoma: demographic, clinical, and pathologic predictors of survival in 1252 cases. Cancer 2006;107:2134-42.

17. Choi YM, Kim TY, Jang EK, Kwon H, Jeon MJ, Kim WG, et al. Standardized thyroid cancer mortality in Korea between 1985 and 2010. Endocrinol Metab (Seoul) 2014;29:530-5.

18. La Vecchia C, Malvezzi M, Bosetti C, Garavello W, Bertuccio $\mathrm{P}$, Levi $\mathrm{F}$, et al. Thyroid cancer mortality and incidence: a global overview. Int J Cancer 2015;136:2187-95.

19. Jung KY, Kim SM, Yoo WS, Kim BW, Lee YS, Kim KW, et al. Postoperative biochemical remission of serum calcitonin is the best predictive factor for recurrence-free survival of medullary thyroid cancer: a large-scale retrospective analysis over 30 years. Clin Endocrinol (Oxf) 2016;84:587-97.

20. Kazaure HS, Roman SA, Sosa JA. Medullary thyroid microcarcinoma: a population-level analysis of 310 patients. Cancer 2012;118:620-7.

21. Cheol Seong S, Kim YY, Khang YH, Heon Park J, Kang HJ, Lee $\mathrm{H}$, et al. Data resource profile: the national health information database of the national health insurance service in South Korea. Int J Epidemiol 2017;46:799-800.

22. World Health Organization. ICD-10: international statistical classification of diseases and related health problems: tenth revision. Gevena: World Health Organization, 2004.

23. Jung KY, Kim SM, Kim MJ, Cho SW, Kim BW, Lee YS, et al. Genotypic characteristics and their association with phenotypic characteristics of hereditary medullary thyroid carcinoma in Korea. Surgery 2018;164:312-8.

24. Kim BH. Registry for medullary thyroid carcinoma in Korea. 2015 Annual Symposium on Science, Technology, and Education \& Autumn Symposium of KES; 2015 Oct 29-31; Busan, KR.

25. Jameson JL. Harrison's principles of internal medicine. 20th ed. New York: McGraw Hill; 2018.
26. KOrean Statistical Information Service. Population statistics based on resident registration [Internet]. Daejeon: KOSIS, 2020 [cited 2020 Oct 19]. Available from: http://kosis.kr/ index/index.do.

27. Korea Central Cancer Registry, National Cancer Center. Annual report of cancer statistics in Korea in 2016 [Internet]. Sejong: Ministry of Health and Welfare, 2018 [cited 2020 Oct 19]. Available from: https://ncc.re.kr/main.ncc?uri=english/ sub04_Statistics.

28. Mathiesen JS, Kroustrup JP, Vestergaard P, Stochholm K, Poulsen PL, Rasmussen AK, et al. Incidence and prevalence of sporadic and hereditary MTC in Denmark 1960-2014: a nationwide study. Endocr Connect 2018;7:829-39.

29. Modigliani E, Cohen R, Campos JM, Conte-Devolx B, Maes B, Boneu A, et al. Prognostic factors for survival and for biochemical cure in medullary thyroid carcinoma: results in 899 patients. The GETC Study Group. Groupe d'étude des tumeurs à calcitonine. Clin Endocrinol (Oxf) 1998;48:265-73.

30. Kim JH, Pyo JS, Cho WJ. Clinicopathological significance and prognosis of medullary thyroid microcarcinoma: a meta-analysis. World J Surg 2017;41:2551-8.

31. Hirsch D, Twito O, Levy S, Bachar G, Robenshtok E, Gross DJ, et al. Temporal trends in the presentation, treatment, and outcome of medullary thyroid carcinoma: an Israeli multicenter study. Thyroid 2018;28:369-76.

32. Cho BY, Choi HS, Park YJ, Lim JA, Ahn HY, Lee EK, et al. Changes in the clinicopathological characteristics and outcomes of thyroid cancer in Korea over the past four decades. Thyroid 2013;23:797-804.

33. Choi JB, Lee SG, Kim MJ, Kim TH, Ban EJ, Lee CR, et al. Dynamic risk stratification in medullary thyroid carcinoma: single institution experiences. Medicine (Baltimore) 2018; 97:e9686.

34. Meijer JA, Bakker LE, Valk GD, de Herder WW, de Wilt JH, Netea-Maier RT, et al. Radioactive iodine in the treatment of medullary thyroid carcinoma: a controlled multicenter study. Eur J Endocrinol 2013;168:779-86. 